OPEN ACCESS

Edited by:

Fan Feng,

The 302th Hospital of PLA, China

Reviewed by:

Yingshi Zhang,

Shenyang Pharmaceutical University,

China

Xudong Gao,

Fifth Medical Center of the PLA

General Hospital, China

*Correspondence:

Dong Dai

dongdai@tmu.edu.cn

Pengfei Liu

liupengfeitj@163.com

Specialty section:

This article was submitted to Molecular and Cellular Oncology,

a section of the journal

Frontiers in Oncology

Received: 30 June 2021

Accepted: 19 August 2021

Published: 17 September 2021

Citation:

Cui Y, Li H, Zhan H, Han T, Dong Y, Tian C, Guo Y, Yan F, Dai D and Liu P (2021) Identification of

Potential Biomarkers for Liver

Cancer Through Gene Mutation

and Clinical Characteristics.

Front. Oncol. 11:733478.

doi: 10.3389/fonc.2021.733478

\section{Identification of Potential Biomarkers for Liver Cancer Through Gene Mutation and Clinical Characteristics}

\author{
Yunlong Cui ${ }^{1}$, Hua $L_{i}{ }^{2}$, Hongjie Zhan ${ }^{3}$, Tao Han ${ }^{4}$, Yixuan Dong ${ }^{5}$, Caijuan Tian ${ }^{6}$, \\ Yixian Guo ${ }^{6}$, Fang Yan $^{6}$, Dong Dai ${ }^{7 *}$ and Pengfei Liu ${ }^{8 *}$ \\ ${ }^{1}$ Department of Hepatobiliary Oncology, National Clinical Research Center for Cancer, Key Laboratory of Cancer Prevention \\ and Therapy of Tianjin, Tianjin's Clinical Research Center for Cancer, Tianjin Medical University Cancer Institute and Hospital, \\ Tianjin, China, ${ }^{2}$ Department of Endoscopy, Tianjin Medical University Cancer Institute and Hospital, National Clinical \\ Research Center for Cancer, Key Laboratory of Cancer Prevention and Therapy of Tianjin, Tianjin's Clinical Research Center \\ for Cancer, Tianjin, China, ${ }^{3}$ Department of Gastric Cancer, National Clinical Research Center for Cancer, Key Laboratory of \\ Cancer Prevention and Therapy of Tianjin, Tianjin's Clinical Research Center for Cancer, Tianjin Medical University Cancer \\ Institute and Hospital, Tianiin, China, ${ }^{4}$ College of Engineering, Peking University, Beijing. China, ${ }^{5}$ Graduate School, Tianjin \\ Academy of Traditional Chinese Medicine, Tianjin, China, ${ }^{6}$ Tianjin Marvel Medical Laboratory, Tianjin Marvelbio Technology \\ Co., Ltd., Tianjin, China, ${ }^{7}$ Department of Molecular Imaging and Nuclear Medicine, National Clinical Research Center for \\ Cancer, Key Laboratory of Cancer Prevention and Therapy of Tianjin, Tianjin's Clinical Research Center for Cancer, Tianjin \\ Medical University Cancer Institute and Hospital, Tianjin, China, ${ }^{8}$ Department of Oncology, Tianjin Academy of Traditional \\ Chinese Medicine Affiliated Hospital, Tianjiin, China
}

Liver cancer is a common malignant tumor worldwide, which is a serious threat to the health of people. We try to investigate some mutations and clinical indicators as candidate markers for the development of liver cancer through targeted region capture technology combined with next-generation sequencing. We collected peripheral blood and liver cancer tissue samples from 32 liver patients concurrently. The SeqCap EZ Prime Choice Probe was used to perform the targeted enrichment; this probe captures 1,000 known cancer-associated genes. We calculated the tumor mutation burden (TMB) for each patient. The high-frequency mutations and these relative genes were identified. Eventually, survival analysis was performed based on the mutations and clinical indicators. In 32 liver patients, a total of 29 high-frequency mutations were investigated. They were located in 25 genes, which were enriched in 9 cellular components (CCs), 6 molecular functions (MFs), and 21 biological processes (BPs). Among them, EZH2 c.1544A>G and CCND1 c.839A>T had the highest mutation frequency (5/32). In the protein-protein interaction (PPI) network, EZH2-DNMT3A, NOTCH1-CCND1, and ABL1-CCND1 were the top three pairs. The survival analysis showed that there were significant differences in progression-free survival (PFS) and overall survival (OS) between the Karnofsky performance score (KPS) groups. The PFS and OS in the TMB high group were higher than those in the TMB low group. OS and tumor stage had a remarkable relationship. In conclusion, EZH2 c.1544A $>\mathrm{G}$ and CCND1 c.839A $>$ T might be potential biomarkers of liver cancer. TMB might be used as a prognosis and survival indicator of liver cancer.

Keywords: liver cancer, circulating tumor DNA (ctDNA), gene mutation, tumor mutation burden (TMB), survival analysis 


\section{INTRODUCTION}

Cancer is a common disease that poses a threat to human health and affects people's quality of life. In 2020, liver cancer was one of the cancers with the lowest survival rates (18\%), followed by pancreatic cancer (9\%). The incidence rate of liver cancer is increasing worldwide; according to the latest cancer statistics, liver cancer has the fastest growth rate among male malignant tumors, with an annual growth rate of $2 \%-3 \%$ (1). Liver cancer can be divided into primary and secondary tumors; primary liver cancer (PLC) can be further divided into hepatocellular carcinoma (HCC), intrahepatic cholangiocarcinoma (ICC), and other rare types (2). The pathogenesis and exact molecular mechanism of liver cancer are not clear.

Mutations in some genes are proven to have a certain relationship with the occurrence and progression of liver cancer (3). For example, Huang and his partners (4) investigated that the base mutation in the p53 gene, which could serve as a novel early diagnostic marker for HCC, provided more information when combined with the usual method of HCC diagnosis. Furthermore, Wong et al. (5) examined that somatic mutations at exon 3 of the $\beta$-catenin gene might give rise to inappropriate activation of the Wnt signaling pathway that plays an important role in HCC. Quaas et al. (6) found that analysis of telomerase reverse transcriptase (TERT) promoter mutation might become a diagnostic tool differentiating hepatocellular adenoma (HCA) from welldifferentiated HCC and transforming lesions.

Circulating tumor DNA (ctDNA), possessing the initial tumor mutational signature, is released by the tumor cells into the blood, and it reflects the alterations of the gene from primary cancers (7). For cancer patients in whom acquiring biopsy specimens is difficult, ctDNA has become a potential noninvasive cancer biomarker. Nowadays, researchers have found multiple applications for ctDNA in the cancer field. Many studies have demonstrated that ctDNA can be applied for the early detection (8) and monitoring of the minimal residual disease of cancer (9). For instance, $\mathrm{Xu}$ et al. (10) constructed a diagnostic prediction model of HCC according to the ctDNA methylation markers, and this model exhibited high diagnostic specificity and sensitivity ( $\mathrm{p}<0.001)$. Additionally, ctDNA plays an important role in the evaluation of molecular heterogeneity of overall disease (11) and monitoring of tumor dynamics (12). Ikeda et al. (13) indicated that ctDNA can be used as a marginally invasive alternative method to identify genetic alterations and discover potential molecular therapeutic targets. Furthermore, Cai et al. (14) identified the mutation profiles in biopsy and plasma specimens of HCC patients and found that ctDNA could overcome the heterogeneity of tumor and real-time track the therapeutic responses. Consequently, the clinical application of ctDNA has attracted the attention of more and more researchers. In this study, we systematically analyzed the gene mutations in the liver using targeted region capture combined with nextgeneration sequencing and identified some potential biomarkers for the occurrence and development of liver cancer.

\section{METHODS}

\section{Patients and Sample Collection}

Patients treated at Tianjin Medical University Cancer Institute and Hospital between July 2017 and May 2018 were enrolled. Thirty-two liver patients were collected and named as L01 to L32 successively. Sixteen of the patients were retained from medical records. The ethylene diamine tetraacetic acid (EDTA) tubes were used to collect peripheral blood. Subsequently, the white blood cell and plasma were separated by centrifuging at $2,500 \mathrm{~g}$ for $10 \mathrm{~min}$. To remove any remaining cellular debris, the plasma was further centrifuged at $16,000 \mathrm{~g}$ for $10 \mathrm{~min}$. The plasma supernatant and matched white blood cells were preserved at $-80^{\circ} \mathrm{C}$ separately. In the meantime, the matching tumor tissue samples were gathered. DNA was extracted from tumor tissues and paired white blood cells (control) utilizing the TIANamp Genomic DNA Kit (Tiangen, China). Nevertheless, circulatingfree DNA (cfDNA) was extracted from the plasma by the QIAamp Circulating Nucleic Acid Kit (Qiagen, Germany). This study was approved by the Ethics Review Committee of Tianjin Medical University Cancer Institute and Hospital.

\section{Target Sequencing}

The targeted region enrichment of matched tumor samples and blood was accomplished by the SeqCap EZ Prime Choice Probe (Roche, Switzerland), which captures a certain amount of $1.1 \mathrm{Mb}$ from 1,000 known mutation genes of cancer-related genes. Afterwards, the constructed libraries were sent for targeted sequencing using the Illumina HiSeq Xten sequencer at Beijing Novogene Bioinformatics Technology Co., Ltd. (Beijing, China). Average sequencing depths of the tumor samples and blood genomes were approximately $500 \times$ and 1,000× respectively.

\section{Sequencing Data Analysis}

The original data were filtered, and the adapter contamination sequences and the low-quality sequences were removed. Subsequently, the clean data were mapped to the hg19 reference genome using Burrows-Wheeler Aligner software (BWA) and analyzed to detect the tumor-specific somatic mutation. To acquire valid mutations, the reads that had exceeded two mismatches were discarded during the alignment process. These mutation sites below $200 \times$ in the tumor and below $100 \times$ in white blood cells were filtered out. The supporting reads of each mutation site were $>3$ in the tumor tissue and $\leq 2$ in ctDNA. The concerned statistics of sequencing results are listed in Supplementary Table S1.

\section{Enrichment Analysis}

Gene Ontology (GO) terms and Kyoto Encyclopedia of Genes and Genomes (KEGG) pathway enrichment analyses of selected genes were implemented through the Database for Annotation, Visualization, and Integrated Discovery (DAVID) V6.7 (http:// david.abcc.ncifcrf.gov/). p $<0.05$ was the cutoff standard. 


\section{Construction of the PPI Network}

In order to better understand the effect of the selected genes, we constructed the protein-protein interaction (PPI) network by searching the Search Tool for the Retrieval of Interacting Genes (STRING) database (http://string-db.org/). Whereafter, the network was visualized by Cytoscape software.

\section{Tumor Mutational Burden}

Tumor mutation burden (TMB) refers to the number of somatic, coding base substitutions, and short insertions and deletions mutations that occur in the tumor tissue. TMB was calculated by the number of somatic base substitution or insertions and deletions alterations per megabase $(\mathrm{Mb})$ in the coding region target territory of the test. These known somatic and deleterious mutations needed to be removed before calculating TMB. Then, the value was concluded to the exome or genome as a whole. The cutoff values of TMB in this study were obtained from the optimal critical value calculated by Youden's indexes through receiver operating characteristic (ROC) curves of overall survival (OS) and progression-free survival (PFS).

\section{Correlation Analysis and Survival Analysis}

The clinical data and sequencing results of 16 patients were used for correlation and survival analyses. The Fisher exact test and MannWhitney U-test were used to assess the statistical significance. Survival analysis was completed by Kaplan-Meier analysis. When $\mathrm{p}<0.05$, the results were considered statistically significant.

\section{RESULTS}

\section{Patients' Characteristics}

Most of the liver cancer patients were men (28/32, 87.50\%) with the median age being 53.5 (range, 31-72) years. Among these patients, 15 patients had HCC, 3 patients had ICC, 2 patients had hilar cholangiocarcinoma (HCCA), 3 patients had liver cancer with pulmonary metastasis, and another 9 patients had unknown disease. Because some patients were missing clinical information, only the clinical data of 16 liver cancer patients were analyzed. Table 1 summarizes the clinical characteristics. Thirteen patients were infected with HBV, and 12 patients had liver cirrhosis. The Karnofsky performance score (KPS) of nine patients was 100, and seven patients was $<100$ (Table 1).

\section{The Analysis of High-Frequency Mutations in HCC Patients}

We integrally analyzed the mutation signature in ctDNA and tumor tissue of every patient. Twenty-nine gene mutations were identified as high-frequency mutations that happened in the lowest two samples (Figure 1A). Among them, EZH2 c.1544A $>\mathrm{G}$ and CCND1 c.839A $>$ T had the highest frequency $(n=5)$, and SMO c.332-2A >C, IRS2 c.4012+2T>G, MAP2K4 c.92A >C, MAPK3 c. $1017+2 \mathrm{~T}>\mathrm{G}$, DNMT3A c. $887 \mathrm{~T}>\mathrm{G}$, RARA c.1339A $>$ C, and KMT2C c.7447G $>$ T were detected in three patients; the remaining 20 mutation sites were discovered in two patients. Furthermore, EZH2 c.1544A>G, KMT2C c.7447G>T, ABL1 c. $2986 \mathrm{~A}>\mathrm{C}$, AXL c. $460 \mathrm{~A}>\mathrm{C}, \mathrm{CDH} 1$ c. $383 \mathrm{~A}>\mathrm{C}, \mathrm{MED} 12$ c.6401A >C, ERBB2 c.3307A>C, NTRK1 c.662G>C, BRD4

TABLE 1 | Clinical characteristics of 16 enrolled liver cancer patients.

\begin{tabular}{|c|c|c|c|}
\hline Clinical characteristics & No. of patients & Clinical characteristics & No. of patients \\
\hline Age & & HCV infection & \\
\hline$\leq 52$ & 8 & Yes & 0 \\
\hline$\geq 52$ & 8 & No & 14 \\
\hline Gender & & Unknown & 2 \\
\hline Male & 15 & Other infections (HPV/HIV, etc.) & \\
\hline Female & 1 & Yes & 0 \\
\hline Tumor size & & No & 14 \\
\hline$<8 \mathrm{~cm}$ & 9 & Unknown & 2 \\
\hline$\geq 8 \mathrm{~cm}$ & 6 & Hypertension & \\
\hline Unknown & 1 & Yes & 2 \\
\hline KPS & & No & 12 \\
\hline$<100$ & 7 & Unknown & 2 \\
\hline$=100$ & 9 & Vascular invasion & \\
\hline Tumor stage & & Yes & 6 \\
\hline I stage & 1 & No & 8 \\
\hline I stage & 4 & Unknown & 2 \\
\hline II stage & 8 & Diabetes & \\
\hline III stage & 3 & Yes & 4 \\
\hline Liver cirrhosis & & No & 10 \\
\hline Yes & 12 & Unknown & 2 \\
\hline No & 2 & & \\
\hline Unknown & 2 & & \\
\hline \multicolumn{4}{|l|}{ HBV infection } \\
\hline Yes & 13 & & \\
\hline No & 1 & & \\
\hline Unknown & 2 & & \\
\hline
\end{tabular}

KPS, Karnofsky Performance Score; HBV, hepatitis B virus; HCV, hepatitis C virus; HPV, human papillomavirus; HIV, human immunodeficiency virus. 

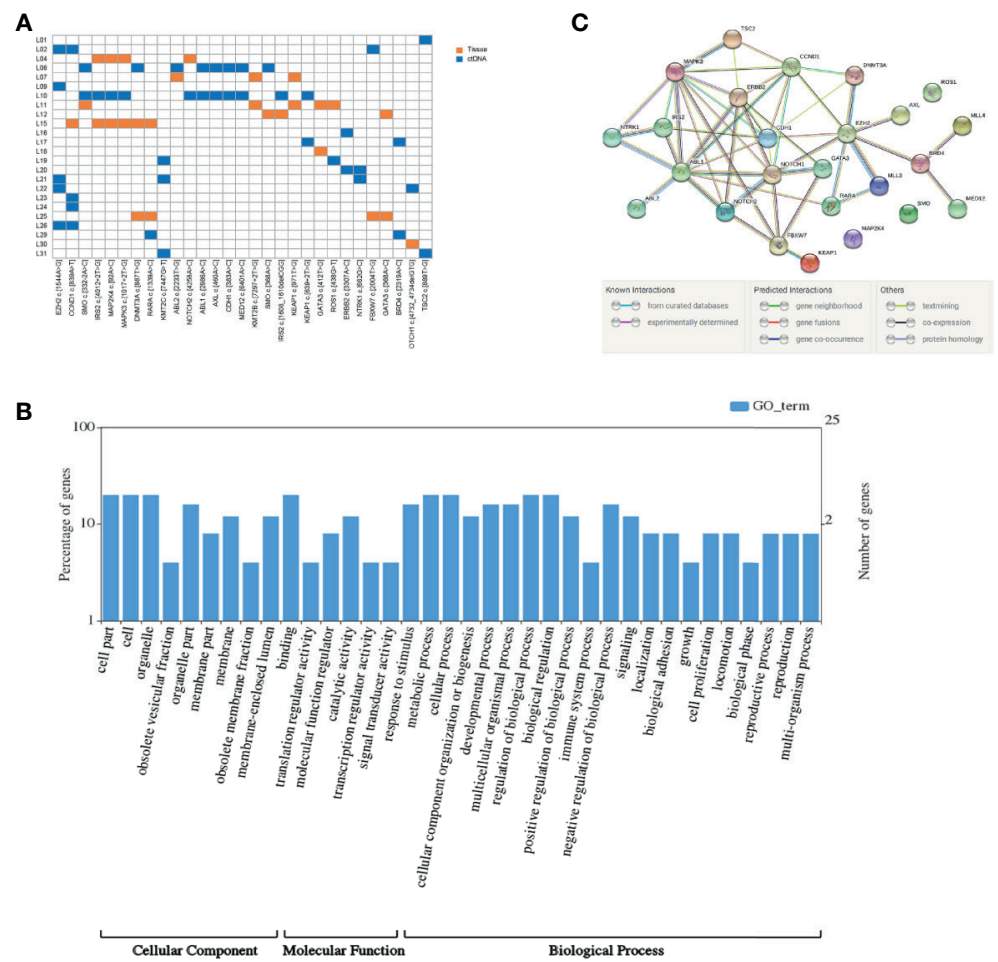

FIGURE 1 | Mutation detection and enrichment of the mutated genes. (A) The number of shared mutations found in two or more patients. Each row represents a patient, and each column represents one mutation. Blue indicates that the mutation hotspot was detected in plasma; orange indicates that the mutation was detected in the tissue. (B) GO enrichment analysis of genes in which 29 high-frequency mutations are located. (C) The PPI networks of these genes in which 29 high-frequency mutations are located.

c.2319A $>$ C, and TSC2 c.889T $>$ G only occurred in ctDNA, and KMT2B c.7297+2T>G, KEAP1 c.971T>G, GATA3 c.412T $>$ G, and GATA3 c.368A $>$ C only occurred in tumor tissue. These 29 highfrequency mutation sites were situated in 25 genes. Among these genes, SMO, KEAP1, IRS2, and GATA3 contained two mutation sites, and the residual 21 genes had only one mutation site.

\section{Enrichment Gene Ontology and Pathway Terms}

KEGG pathway and GO enrichment analyses were performed on the above 25 genes. These genes were found to be enriched in 36 GO terms, including 9 cellular components (CCs), 6 molecular functions (MFs), and 21 biological processes (BPs). Among CCs, the mostly enriched terms were cell part, cell, and organelle. In MF, binding was the only term that was mostly enriched. Metabolic process, cellular process, regulation of biological process, and biological regulation were the mostly enriched BPs (Figure 1B).

\section{The PPI Network}

Based on the STRING analysis, 50 protein-protein interaction pairs of the above 25 genes were identified (Figure 1C). The top 20 pairs that had a high combined score are listed in Table 2. Among them, EZH2-DNMT3A, NOTCH1-CCND1, and ABL1-
CCND1 were the top three pairs with the highest scores, namely, $0.988,0.978$, and 0.973 , respectively.

\section{Assessment of Tumor Mutational Burden}

Based on correlation analysis with clinical data, we used the Youden index to select two TMB cutoff values for grouping: 59 mutations $/ \mathrm{Mb}(\mathrm{AUC}=0.583$, sensitivity $=0.5$, and specificity $=$ 0.8 ), and 79 mutations $/ \mathrm{Mb}(\mathrm{AUC}=0.547$, sensitivity $=0.625$, and specificity $=0.625$ ). In the former, there were 9 patients with $\mathrm{TMB} \leq 59$ and 23 patients with $\mathrm{TMB}>59$. In the latter, there were 13 patients with $\mathrm{TMB} \leq 79$ and 19 patients with $\mathrm{TMB}>79$. When the TMB threshold was 59 and 79, there were significant differences in serum AFP between the low and high group, respectively ( $p=0.002$ and $p=0.001$, Table 3$)$. Although there was no statistical difference in the Kaplan-Meier test in the survival analysis, the survival and recurrence times of the $\mathrm{TMB}>59$ mutations/Mb group were significantly longer than those of the $\mathrm{TMB} \leq 59$ mutations/Mb group according to the survival curve (Figure 2).

\section{Survival Analysis}

There was no statistically significant relationship between PFS and age, diabetes, tumor size, gamma-glutamyl transpeptidase 
TABLE 2 | The top 20 pairs of the PPI network with high combined score.

\begin{tabular}{llc}
\hline Gene1 & Gene2 & Combined score \\
\hline EZH2 & DNMT3A & 0.988 \\
NOTCH1 & CCND1 & 0.978 \\
ABL1 & CCND1 & 0.973 \\
GATA3 & NOTCH1 & 0.969 \\
MAPK3 & TSC2 & 0.965 \\
MAPK3 & CDH1 & 0.961 \\
IRS2 & MAPK3 & 0.950 \\
EZH2 & RARA & 0.946 \\
NTRK1 & IRS2 & 0.944 \\
NTRK1 & ABL1 & 0.939 \\
ABL2 & ABL1 & 0.936 \\
ERBB2 & CCND1 & 0.931 \\
FBXW7 & NOTCH1 & 0.931 \\
DNMT3A & CCND1 & 0.924 \\
EZH2 & MLL3 & 0.924 \\
FBXW7 & KEAP1 & 0.911 \\
NOTCH1 & NOTCH2 & 0.908 \\
MLL3 & RARA & 0.906 \\
NOTCH1 & ERBB2 & 0.901 \\
CDH1 & CCND1 & 0.886 \\
\hline PP1 0 1 & &
\end{tabular}

PPI, protein-protein interaction.

(GGT), alanine aminotransferase (ALT), alkaline phosphatase (ALP), cholinesterase (CHE), or serum albumin (ALB), and OS. The mutations that occur in more than three clinical patients were selected out, namely, CCND1 c.839A >T, EZH2 c.1544A $>\mathrm{G}$, IRS2 c.4012+2T >G, KMT2C c.7447G >T, RARA c.1339A $>$ C, and SMO c.332-2A $>$ C. We did not find a marked relationship between PFS or OS and the presence of these mutations ( $\mathrm{p}=$ 0.273 and 0.295 ). Nevertheless, there was a meaningful positive correlation between KPS score and PFS ( $p<0.001$, Figure $3 \mathrm{~A}$ ) or OS ( $\mathrm{p}<0.001$, Figure 3C). Patients with a higher KPS scores had longer PFS and OS, and all patients with 100 KPS score survived until the end of the study. Besides, PFS scores were significantly different between patients with and without vascular invasion $(\mathrm{p}=0.028$, Figure 3B). Furthermore, stage I and II liver cancer patients have longer OS than those with stages III and IV ( $\mathrm{p}=$ 0.049, Figure 3D).

\section{DISCUSSION}

In this study, we identified the mutations in liver cancer patients by high-throughput sequencing. EZH2 c.1544A > G and CCND1 c.839A $>\mathrm{T}$ were found in five liver cancer patients with the highest mutation frequency. The Enhancer of Zeste Homologue 2 gene (EZH2), a component of polycomb repressive complex 2 (PRC2), is located on human chromosome 7q35. The role of $\mathrm{EZH} 2$ gene in tumors, especially in tumor cell invasion and metastasis, has gradually become a research hotspot. EZH2 gene is highly expressed in a variety of malignant tumors but is low or not expressed in normal tissues (15). EZH2 expression is also associated with the malignant features of the tumor and the poor prognosis of the patient $(16,17)$. Further studies revealed that EZH2 inhibits the expression of ET microRNAs (miRNAs) (EZH2-targeted miRNAs) through the H3K27me3 pathway, thus forming a positive feedback loop of EZH2 miRNAs, maintaining the high expression of EZH2 and promoting the proliferation of tumor cells by regulating key growth inhibitory factors. EZH2 gene participates in the tumorigenesis and progression by promoting cell proliferation, cell cycle arrest, cell migration, and invasion. In addition, EZH2 is the second histone methyltransferase gene that is found to be mutated in cancer. The research found that EZH2 mutations, which cause the change in a single tyrosine in the SET domain of the EZH2 protein (Tyr641), have a high mutation rate in follicular lymphoma (FL), and these mutations were very stable during the development of the disease (18). EZH2 mutation had a certain significance in the development of FL and can represent its early event (19). Studies

TABLE 3 | Correlation between TMB and clinicopathological parameters ( $p$-value).

\begin{tabular}{|c|c|c|c|c|c|c|}
\hline \multirow[t]{2}{*}{ Clinical characteristics } & \multicolumn{3}{|c|}{ TMB threshold: 59/MB } & \multicolumn{3}{|c|}{ TMB threshold: 79/MB } \\
\hline & Low group (0-59/MB) & High group (>59/MB) & p-value & Low group (0-79/MB) & High group (>79/MB) & p-value \\
\hline \multicolumn{7}{|l|}{ Age } \\
\hline$\leq 52$ & 2 & 6 & 1 & 4 & 4 & 1 \\
\hline$>53$ & 3 & 5 & & 4 & 4 & \\
\hline \multicolumn{7}{|l|}{ Liver cirrhosis } \\
\hline Yes & 4 & 8 & 0.401 & 7 & 5 & 0.713 \\
\hline No & 1 & 1 & & 1 & 1 & \\
\hline \multicolumn{7}{|l|}{ Tumor size } \\
\hline$<8 \mathrm{~cm}$ & 3 & 6 & 0.604 & 5 & 4 & 0.608 \\
\hline$\geq 8 \mathrm{~cm}$ & 1 & 5 & & 2 & 4 & \\
\hline \multicolumn{7}{|l|}{ Tumor stage } \\
\hline I, II stage & 1 & 4 & 1 & 2 & 3 & 1 \\
\hline III, IV stage & 4 & 7 & & 6 & 5 & \\
\hline \multicolumn{7}{|l|}{ KPS } \\
\hline$<100$ & 3 & 4 & 0.596 & 4 & 3 & 1 \\
\hline$=100$ & 2 & 7 & & 4 & 5 & \\
\hline AFP & 5 & 11 & $0.002^{\star \star}$ & 8 & 8 & $0.001^{* *}$ \\
\hline
\end{tabular}

TMB, tumor mutational burden; KPS, Karnofsky Performance Score; AFP, alpha fetoprotein. ${ }^{* *} p<0.001$. 

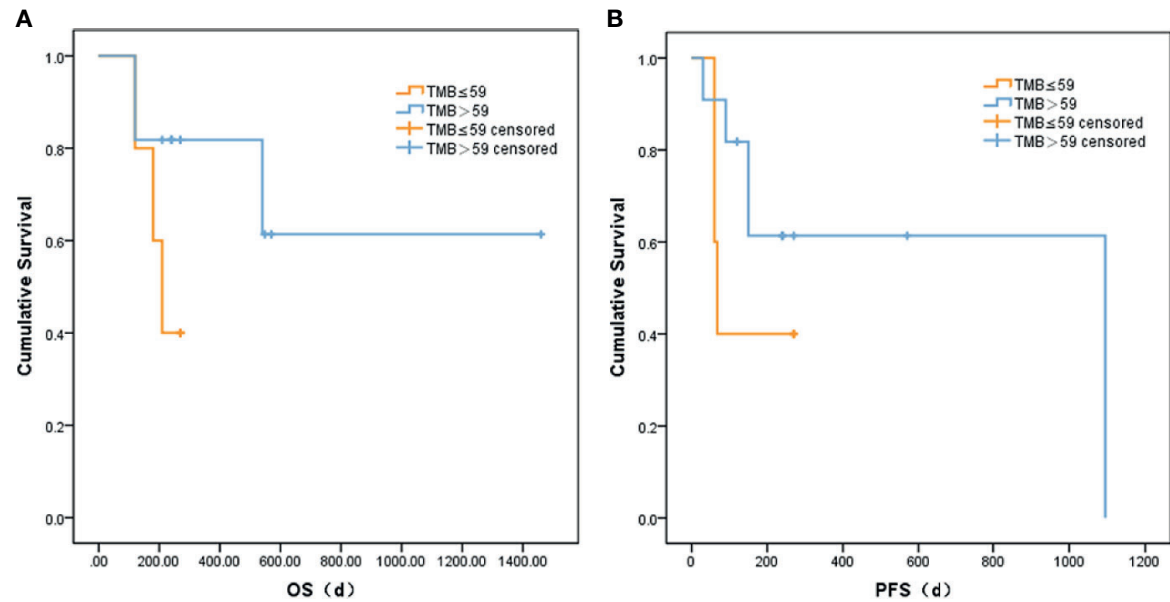

FIGURE 2 | Comparison of (A) OS and (B) PFS between liver cancer patients with different TMB.

also found frequent mutations in the EZH2 SET domain [for example, tyrosine residue 646 (Y646)] and EZH2 somatic mutations in various tumors such as germinal center (GC) diffuse large B-cell lymphoma (DLBCL), non-small cell lung cancer, prostate cancer, and colon carcinoma (20). These findings indicate that EZH2 may be a potential therapeutic target in malignant tumors, including liver cancer. Human CCND1 gene encodes the cyclin D1 protein, a regulator of cyclin-dependent kinases (CDKs). At present, CCND1 gene has been recognized as a proto-oncogene whose overexpression can lead to uncontrolled cell proliferation and malignant changes. The CCND1 gene mutation also has been implicated in the development and progression of various cancers. In lung cancer, CCND1 mutation was significantly associated with pathological types and smoking (21). Grünhage et al. (22) indicated that CCND1 c.870A $>\mathrm{G}$ mutation was associated with familial colorectal cancer. Although there is no literature report on the impact of $\mathrm{EZH} 2$ c.1544A $>\mathrm{G}$ and CCND1 c.839A $>\mathrm{T}$ on liver cancer, these mutations are pathogenic, and the scores were 0.99 and 0.94 respectively according to the Catalogue of Somatic Mutations in Cancer (COSMIC) database (https://cancer.sanger. ac.uk/cosmic). This indicated that these two mutations have some significance in the study of hepatocellular carcinogenesis.

In order to better understand the interaction between the selected genes, we conducted PPI analysis based on the STRING database. According to the combined score, EZH2-DNMT3A, NOTCH1-CCND1, and ABL1-CCND1 ranked in the top 3 with the scores of $0.988,0.978$, and 0.973 , respectively. EZH2 is an element of the epigenetic regulator PRC2 that inhibits gene expression (23). Overexpression of EZH2 is a common case in human cancers and is linked with tumor progression and poor prognosis (12). DNA methylation, one of the most important epigenetic modifications, is essential to gene expression regulation, genomic imprinting, $\mathrm{X}$ chromosome inactivation, and tumorigenesis (24). In mammals, DNA methylation patterns are written and regulated by DNA methyltransferases (DNMTs), including DNMT1, DNMT3A, and DNMT3B. Epigenetic damage caused by abnormal DNMTs is related to tumorigenesis, and EZH2 can serve as a recruitment platform for DNA DNMTs. Our result based on the STRING database also showed that there were known interactions and other relationships between EZH2 and DNMT3A. NOTCH proteins (NOTCH1, NOTCH2, NOTCH3, and NOTCH4) play vital roles in embryonic development. Mounting studies indicate that $\mathrm{NOTCH}$ is conducive to the pathogenesis of hematopoietic and solid malignancies $(25,26)$. NOTCH1 mainly engages in angiogenesis and regulates endothelial cell proliferation and migration. The ABL1 proto-oncogene encodes a cytoplasmic and nuclear protein tyrosine kinase that is involved in the processes of cell differentiation, cell adhesion, cytokinesis, and stress response. ABL1 was first discovered as the oncogene in the Abelson murine leukemia virus in the last 38 years (27) and was later recognized as an oncogene participating in chromosomal translocations in human leukemia. The function of CCND1 has been introduced in the previous paragraph. Our result verified that there are clear interactions between NOTCH1 and CCND1, and ABL1 and CCND1 based on data collated from the KEGG databases and others. However, the specific correlations of these genes need to be further studied in subsequent research.

TMB has been regarded as an emerging and isolated biomarker of outcomes with immunotherapy in a variety of tumor types. For instance, Friedlaender et al. (28) showed that plasma TMB could be a dynamic biomarker for immunotherapy treatment in non-small-cell lung cancer (NSCLC). Devarakonda et al. (29) investigated that there was an association between high non-synonymous TMB and the better prognosis in patients with resected NSCLC. Due to the different TMB calculation methods, related thresholds, cancer types, and sequencing panels, there is 
A

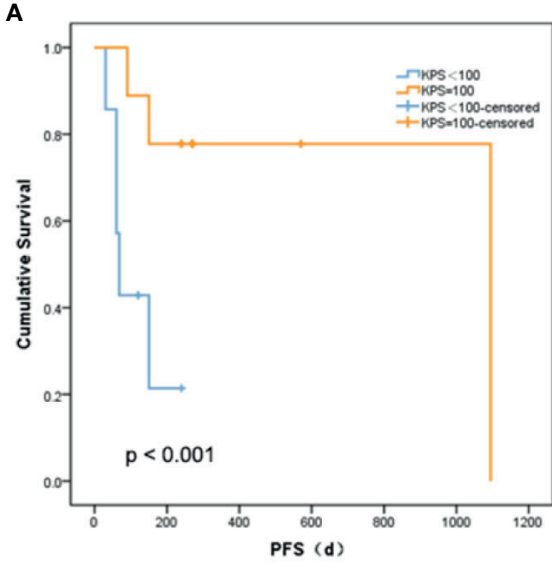

C

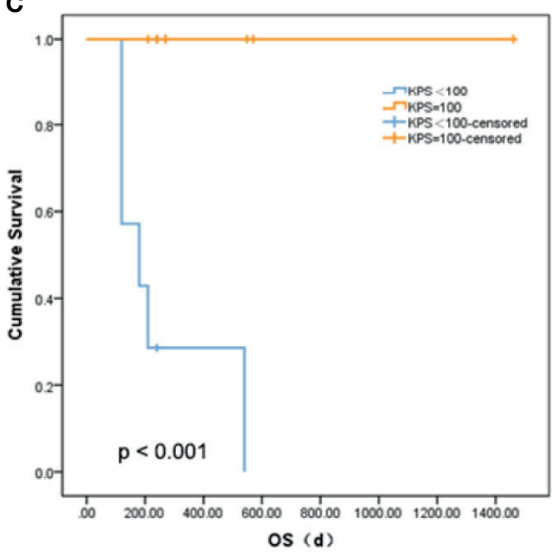

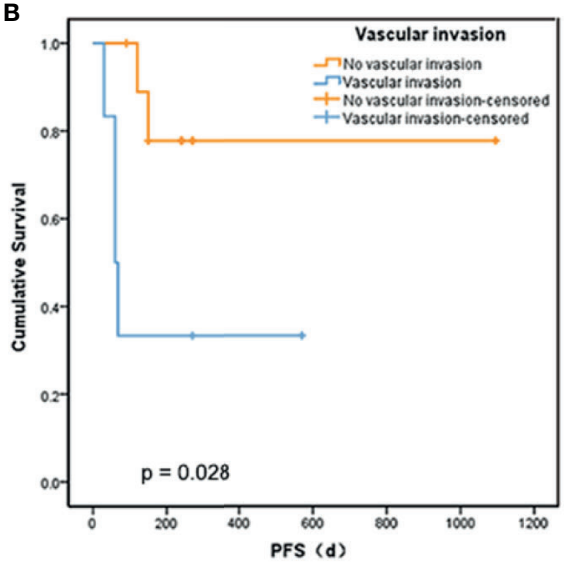

D

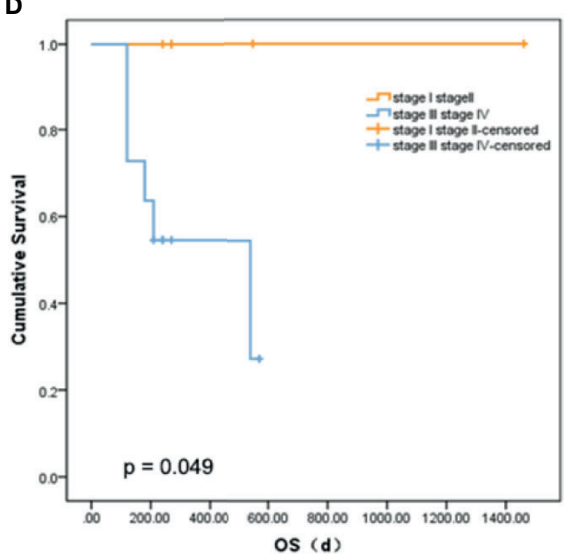

FIGURE 3 | Relationship between survival and clinical indicators. (A) Comparison of PFS between liver cancer patients with different KPS. (B) Comparison of PFS between liver cancer patients with different vascular invasion status. (C) Comparison of OS between liver cancer patients with different KPS. (D) Comparison of OS between liver cancer patients with different tumor stage.

no uniform division method in the related research fields, and there is less TMB-related literature on liver cancer. The TMB filtration criteria in our study were relatively rough, so the TMB values obtained were high. In the research, the ROC curve based on TMB and OS during follow-up indicated that the best cutoff point was 59 mutations/Mb. Based on the ROC curve of TMB and PFS, the best cutoff point was 79 mutations/Mb. Therefore, we chose 59 and 79 as critical points for grouping and subsequent analysis. Regardless of the critical point of 59 or 79 , except for AFP, most of the clinical indicators were not significantly different between the TMB low group and the TMB high group. Moreover, the high-frequency mutations have been shown to be unrelated to patients' survival. There might be two reasons to explain it. One reason is in the small sample size and the missing clinical information of some patients; only 16 liver cancer patients were further analyzed. The other reason is that the treatments of patients were different. Among the 16 patients, 3 patients underwent radical resection, 3 interventional treatment, 2 radiofrequency therapy, 2 surgery, and the other patients a combination of multiple methods. Our study identified that people with higher TMB had longer overall survival and a better prognosis than those with lower TMB. This meant that the mutated genes and TMB may also act as a biomarker in the development and treatment of liver cancer. However, because the sample size was small in our study, together with the selection of the TMB critical point, further validation of this conclusion is needed in subsequent studies.

The main limitation of this study is that a low number of patients were included; meanwhile, due to the retrospective analysis, clinical information and survival data in some patients are missing, resulting in fewer patients in the survival analysis. Therefore, more cases, biochemical indicators, and additional molecular/cellular experiments are required for a prospective study to verify the results in the future. Hence, although we obtained meaningful results from this study, further research is still necessary. 


\section{CONCLUSION}

Overall, EZH2 c.1544A >G and CCND1 c.839A >T might be novel potential biomarkers of liver cancer. The TMB high group had longer OS or PFS than the TMB low group. Further in-depth studies with large sample sizes are progressing gradually to verify the results and explore their mechanisms on the development of liver cancer.

\section{DATA AVAILABILITY STATEMENT}

The data presented in the study are deposited in the NCBI database repository, accession number PRJNA756802. Further inquiries can be directed to the corresponding authors.

\section{ETHICS STATEMENT}

The studies involving human participants were reviewed and approved by Tianjin Medical University Cancer Institute and Hospital. The patients/participants provided their written informed consent to participate in this study.

\section{REFERENCES}

1. Sung H, Ferlay J, Siegel RL, Laversanne M, Soerjomataram I, Jemal A, et al. Global Cancer Statistics 2020: GLOBACAN Estimates of Incidence and Mortality Worldwide for 36 Cancers in 185 Countries. CA Cancer J Clin (2021) 71(3):209-49. doi: 10.3322/caac.21660

2. Wang G, Wang Q, Liang N, Xue H, Yang T, Chen X, et al. Oncogenic Driver Genes and Tumor Microenvironment Determine the Type of Liver Cancer. Cell Death Dis (2020) 11:313. doi: 10.1038/s41419-020-2509-x

3. Calderaro J, Ziol M, Paradis V, Zucman-Rossi J. Molecular and Histological Correlations in Liver Cancer. J Hepatol (2019) 71:616-30. doi: 10.1016/ j.jhep.2019.06.001

4. Huang XH, Sun LH, Lu DD, Sun Y, Ma LJ, Zhang XR, et al. Codon 249 Mutation in Exon 7 of P53 Gene in Plasma DNA: Maybe a New Early Diagnostic Marker of Hepatocellular Carcinoma in Qidong Risk Area, China. World J Gastroenterol (2003) 9:692-5. doi: 10.3748/wjg.v9.i4.692

5. Wong CM, Fan ST, Ng IOL. $\beta$-Catenin Mutation and Overexpression in Hepatocellular Carcinoma: Clinicopathologic and Prognostic Significance. Cancer (2001) 92(1):136-45. doi: 10.1002/1097-0142(20010701)92:1<136:: aid-cncr $1301>3.0 . c 0 ; 2-r$

6. Quaas A, Oldopp T, Tharun L, Kingenfeld C, Krech T, Sauter G, et al. Frequency of TERT Promoter Mutations in Primary Tumors of the Liver. Virchows Arch (2014) 465:673-7. doi: 10.1007/s00428-014-1658-7

7. Gao J, Xi L, Yu R, Xu H, Wu M, Huang H. Differential Mutation Detection Capability Through Capture-Based Targeted Sequencing in Plasma Samples in Hepatocellular Carcinoma. Front Oncol (2021) 11:596789. doi: 10.3389/ fonc.2021.596789

8. Madhavan D, Wallwiener M, Bents K, Zucknick M, Nees J, Schott S, et al. Plasma DNA Integrity as a Biomarker for Primary and Metastatic Breast Cancer and Potential Marker for Early Diagnosis. Breast Cancer Res Treat (2014) 146:163-74. doi: 10.1007/s10549-014-2946-2

9. McBride DJ, Orpana AK, Sotiriou C, Joensuu H, Stephens PJ, Mudie LJ, et al. Use of Cancer-Specific Genomic Rearrangements to Quantify Disease Burden in Plasma From Patients With Solid Tumors. Genes Chromosomes Cancer (2010) 49:1062-9. doi: 10.1002/gcc.20815

10. Xu R, Wei W, Krawczyk M, Wang W, Luo H, Flagg K, et al. Circulating Tumour DNA Methylation Markers for Diagnosis and Prognosis of Hepatocellular Carcinoma. Nat Mater (2017) 16:1155-61. doi: 10.1038/ nmat4997

\section{AUTHOR CONTRIBUTIONS}

YC, DD, and PL designed the study and helped in preparing the manuscript. HL, HZ, TH, and YD conducted the data mining and collection. CT, YG, and FY conducted the bioinformatics analyses and wrote the manuscript. All authors contributed to the article and approved the submitted version.

\section{FUNDING}

This study was supported by the Tianjin Natural Science Foundation (grant no. 17JCYBJC25100) and the National Natural Science Foundation of China (grant no. J2024008).

\section{SUPPLEMENTARY MATERIAL}

The Supplementary Material for this article can be found online at: https://www.frontiersin.org/articles/10.3389/fonc.2021. 733478/full\#supplementary-material

11. Chan KC, Jiang P, Chan CW, Sun K, Wong J, Hui EP, et al. Noninvasive Detection of Cancer-Associated Genome-Wide Hypomethylation and Copy Number Aberrations by Plasma DNA Bisulfite Sequencing. Proc Natl Acad Sci USA (2013) 110:18761-8. doi: 10.1073/pnas.1313995110

12. Heitzer E, Ulz P, Belic J, Gutschi S, Quehenberger F, Fischereder K, et al. Tumor-Associated Copy Number Changes in the Circulation of Patients With Prostate Cancer Identified Through Whole-Genome Sequencing. Genome Med (2013) 5:30. doi: 10.1186/gm434

13. Ikeda S, Lim JS, Kurzrock R. Analysis of Tissue and Circulating Tumor DNA by Next Generation Sequencing of Hepatocellular Carcinoma: Implications for Targeted Therapeutics. Mol Cancer Ther (2018) 17:1114-22. doi: 10.1158/ 1535-7163.MCT-17-0604

14. Cai ZX, Chen G, Zeng YY, Dong XQ, Lin MJ, Huang XH, et al. Circulating Tumor DNA Profiling Reveals Clonal Evolution and Real-Time Disease Progression in Advanced Hepatocellular Carcinoma. Int J Cancer (2017) 141:977-85. doi: 10.1002/ijc.30798

15. Duan R, Du W, Guo W. EZH2: A Novel Target for Cancer Treatment. J Hematol Oncol (2020) 13:104. doi: 10.1186/s13045-020-00937-8

16. Liu T, Cai J, Cai J, Wang Z, Cai L. EZH2-miRNA Positive Feedback Promotes Tumor Growth in Ovarian Cancer. Front Oncol (2021) 10:608393. doi: $10.3389 /$ fonc. 2020.608393

17. Zhang Y, Liu G, Lin C, Liao G, Tang B. Silencing the EZH2 Gene by RNA Interference Reverses the Drug Resistance of Human Hepatic MultidrugResistant Cancer Cells to 5-Fu. Life Sci (2013) 92:896-902. doi: 10.1016/ j.lfs.2013.03.010

18. Morin RD, Johnson NA, Severson TM, Mungall AJ, An J, Goya R, et al. Somatic Mutations Altering EZH2 (Tyr641) in Follicular and Diffuse Large BCell Lymphomas of Germinal-Center Origin. Nat Genet (2010) 42:181-5. doi: 10.1038/ng.518

19. Bödör C, Grossmann V, Popov N, Okosun J, O'Riain C, Tan K, et al. EZH2 Mutations are Frequent and Represent an Early Event in Follicular Lymphoma. Blood (2013) 122:3165-8. doi: 10.1182/blood-2013-04-496893

20. Souroullas GP, Jeck WR, Parker JS, Simon JM, Liu JY, Paulk J, et al. An Oncogenic Ezh2 Mutation Induces Tumors Through Global Redistribution of Histone 3 Lysine 27 Trimethylation. Nat Med (2016) 22:632-40. doi: 10.1038/nm.4092

21. Shang Y, Li X, Liu W, Shi X, Yuan S, Huo R, et al. Comprehensive Genomic Profile of Chinese Lung Cancer Patients and Mutation Characteristics of Individuals Resistant to Icotinib/Gefitinib. Sci Rep (2020) 10:20243. doi: 10.1038/s41598-020-76791-y 
22. Grünhage F, Jungck M, Lamberti C, Berg C, Becker U, Schulte-Witte H, et al. Association of Familial Colorectal Cancer With Variants in the E-Cadherin (CDH1) and Cyclin D1 (CCND1) Genes. Int J Colorectal Dis (2008) 23 (2):147-54. doi: 10.1007/s00384-007-0388-6

23. Sauvageau M, Sauvageau G. Polycomb Group Proteins: Multi-Faceted Regulators of Somatic Stem Cells and Cancer. Cell Stem Cell (2010) 7 (3):299-313. doi: 10.1016/j.stem.2010.08.002

24. Mahmood N, Rabbani SA. DNA Methylation Readers and Cancer: Mechanistic and Therapeutic Applications. Front Oncol (2019) 9:489. doi: 10.3389/fonc.2019.00489

25. Lomeli H, Castillo-Castellanos F. Notch Signaling and the Emergence of Hematopoietic Stem Cells. Dev Dyn (2020) 249(11):1302-17. doi: 10.1002/ dvdy. 230

26. Fasoulakis Z, Daskalakis G, Theodora M, Antsaklis P, Sindos M, Diakosavvas $\mathrm{M}$, et al. The Relevance of Notch Signaling in Cancer Progression. Adv Exp Med Biol (2021) 1287:169-81. doi: 10.1007/978-3-030-55031-8_11

27. Goff SP, Gilboa E, Witte ON, Baltimore D. Structure of the Abelson Murine Leukemia Virus Genome and the Homologous Cellular Gene: Studies With Cloned Viral DNA. Cell (1980) 22(3):777-85. doi: 10.1016/0092-8674(80) 90554-1

28. Friedlaender A, Nouspikel T, Christinat Y, Ho L, McKee T, Addeo A. TissuePlasma TMB Comparison and Plasma TMB Monitoring in Patients With Metastatic Non-Small Cell Lung Cancer Receiving Immune Checkpoint Inhibitors. Front Oncol (2020) 10:142. doi: 10.3389/fonc.2020.00142
29. Devarakonda S, Rotolo F, Tsao MS, Lanc I, Brambilla E, Masood A, et al. Tumor Mutation Burden as a Biomarker in Resected Non-Small-Cell Lung Cancer. J Clin Oncol (2018) 36(30):2995-3006. doi: 10.1200/JCO.2018.78.1963

Conflict of Interest: Author CT, YG and FY were employed by the company Tianjin Marvelbio Technology Co., Ltd.

The remaining authors declare that the research was conducted in the absence of any commercial or financial relationships that could be construed as a potential conflict of interest.

Publisher's Note: All claims expressed in this article are solely those of the authors and do not necessarily represent those of their affiliated organizations, or those of the publisher, the editors and the reviewers. Any product that may be evaluated in this article, or claim that may be made by its manufacturer, is not guaranteed or endorsed by the publisher.

Copyright (C) 2021 Cui, Li, Zhan, Han, Dong, Tian, Guo, Yan, Dai and Liu. This is an open-access article distributed under the terms of the Creative Commons Attribution License (CC BY). The use, distribution or reproduction in other forums is permitted, provided the original author(s) and the copyright owner(s) are credited and that the original publication in this journal is cited, in accordance with accepted academic practice. No use, distribution or reproduction is permitted which does not comply with these terms. 